\title{
DISCOVERY OF A NEW TeV GAMMA-RAY SOURCE: VER J0521+211
}

S. Archambault ${ }^{1}$, T. Arlen ${ }^{2}$, T. Aune ${ }^{2}$, B. Behera ${ }^{3}$, M. Beilicke ${ }^{4}$, W. Benbow ${ }^{5}$, R. Bird ${ }^{6}$, A. Bouvier ${ }^{7}$, J. H. Buckley ${ }^{4}$,

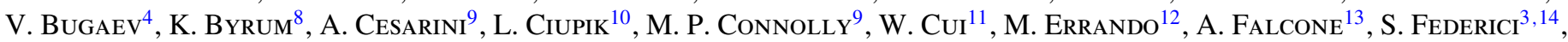

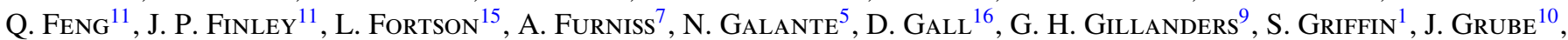

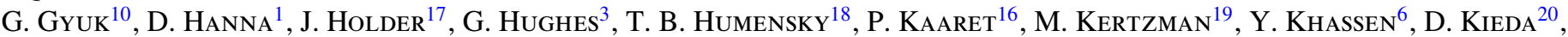

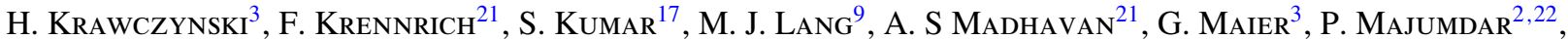
S. McArthur ${ }^{23}$, A. McCann ${ }^{24}$, J. Millis ${ }^{25}$, P. Moriarty ${ }^{26}$, R. Mukherjee $^{12}$, A. O'Faoláin de Bhróithe ${ }^{6}$, R. A. Ong ${ }^{2}$,

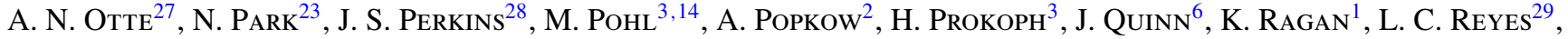

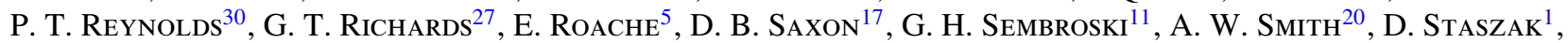
I. Telezhinsky ${ }^{3,14}$, M. Theiling ${ }^{11}$, A. Varlotta ${ }^{11}$, V. V. Vassiliev ${ }^{2}$, S. Vincent ${ }^{3}$, S. P. Wakely ${ }^{23}$, T. C. Weekes ${ }^{5}$, A. Weinstein ${ }^{21}$, R. Welsing ${ }^{3}$, D. A. Williams ${ }^{7}$, B. ZITZER ${ }^{8}$

(The VERITAS COLlaboration)

AND

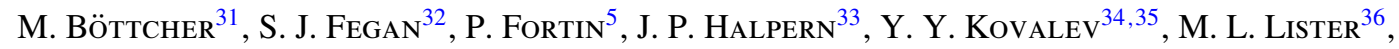
J. LiU ${ }^{33}$, A. B. PushKareV ${ }^{37,38}$, AND P. S. SMith ${ }^{39}$

${ }^{1}$ Physics Department, McGill University, Montreal, QC H3A 2T8, Canada

${ }^{2}$ Department of Physics and Astronomy, University of California, Los Angeles, CA 90095, USA ${ }^{3}$ DESY, Platanenallee 6, D-15738 Zeuthen, Germany

${ }^{4}$ Department of Physics, Washington University, St. Louis, MO 63130, USA

${ }^{5}$ Fred Lawrence Whipple Observatory, Harvard-Smithsonian Center for Astrophysics, Amado, AZ 85645, USA; fortin@ veritas.sao.arizona.edu

${ }^{6}$ School of Physics, University College Dublin, Belfield, Dublin 4, Ireland

${ }^{7}$ Santa Cruz Institute for Particle Physics and Department of Physics, University of California, Santa Cruz, CA 95064, USA

${ }^{8}$ Argonne National Laboratory, 9700 S. Cass Avenue, Argonne, IL 60439, USA

${ }^{9}$ School of Physics, National University of Ireland Galway, University Road, Galway, Ireland

${ }^{10}$ Astronomy Department, Adler Planetarium and Astronomy Museum, Chicago, IL 60605, USA

${ }^{11}$ Department of Physics, Purdue University, West Lafayette, IN 47907, USA

${ }^{12}$ Department of Physics and Astronomy, Barnard College, Columbia University, NY 10027, USA; errando@astro.columbia.edu

${ }^{13}$ Department of Astronomy and Astrophysics, 525 Davey Lab, Pennsylvania State University, University Park, PA 16802, USA

${ }^{14}$ Institute of Physics and Astronomy, University of Potsdam, D-14476 Potsdam-Golm, Germany

${ }^{15}$ School of Physics and Astronomy, University of Minnesota, Minneapolis, MN 55455, USA

${ }^{16}$ Department of Physics and Astronomy, University of Iowa, Van Allen Hall, Iowa City, IA 52242, USA

${ }^{17}$ Department of Physics and Astronomy and the Bartol Research Institute, University of Delaware, Newark, DE 19716, USA; jholder@physics.udel.edu

${ }^{18}$ Physics Department, Columbia University, New York, NY 10027, USA

${ }^{19}$ Department of Physics and Astronomy, DePauw University, Greencastle, IN 46135-0037, USA

${ }^{20}$ Department of Physics and Astronomy, University of Utah, Salt Lake City, UT 84112, USA

21 Department of Physics and Astronomy, Iowa State University, Ames, IA 50011, USA

${ }^{22}$ Saha Institute of Nuclear Physics, Kolkata 700064, India

${ }^{23}$ Enrico Fermi Institute, University of Chicago, Chicago, IL 60637, USA

${ }^{24}$ Kavli Institute for Cosmological Physics, University of Chicago, Chicago, IL 60637, USA

${ }^{25}$ Department of Physics, Anderson University, 1100 East 5th Street, Anderson, IN 46012, USA

${ }^{26}$ Department of Life and Physical Sciences, Galway-Mayo Institute of Technology, Dublin Road, Galway, Ireland

${ }^{27}$ School of Physics and Center for Relativistic Astrophysics, Georgia Institute of Technology, 837 State Street NW, Atlanta, GA 30332-0430, USA

${ }^{28}$ NASA/Goddard Space-Flight Center, Code 661, Greenbelt, MD 20771, USA

${ }^{29}$ Physics Department, California Polytechnic State University, San Luis Obispo, CA 94307, USA

${ }^{30}$ Department of Applied Physics and Instrumentation, Cork Institute of Technology, Bishopstown, Cork, Ireland

${ }^{31}$ Centre for Space Research, North-West University, Potchefstroom 2531, South Africa

${ }^{32}$ Laboratoire Leprince-Ringuet, École polytechnique, CNRS/IN2P3, Palaiseau, France; sfegan@1lr.in2p3.fr

${ }^{33}$ Columbia Astrophysics Laboratory, Columbia University, New York, NY 10027, USA

${ }^{34}$ Max-Planck-Institut für Radioastronomie, Auf dem Hügel 69, D-53121 Bonn, Germany

${ }^{35}$ Astro Space Center of Lebedev Physical Institute, Profsoyuznaya Str. 84/32, 117997 Moscow, Russia

${ }^{36}$ Department of Physics, Purdue University, 525 Northwestern Avenue, West Lafayette, IN 47907, USA

${ }^{37}$ Pulkovo Astronomical Observatory, Pulkovskoe Chaussee 65/1, 196140 St. Petersburg, Russia

${ }^{38}$ Crimean Astrophysical Observatory, 98409 Nauchny, Crimea, Ukraine

${ }^{39}$ Steward Observatory, University of Arizona, Tucson, AZ 85721, USA

Received 2013 June 13; accepted 2013 August 21; published 2013 September 27

\begin{abstract}
We report the detection of a new TeV gamma-ray source, VER J0521+211, based on observations made with the VERITAS imaging atmospheric Cherenkov Telescope Array. These observations were motivated by the discovery of a cluster of $>30 \mathrm{GeV}$ photons in the first year of Fermi Large Area Telescope observations. VER J0521+211 is relatively bright at $\mathrm{TeV}$ energies, with a mean photon flux of $\left(1.93 \pm 0.13_{\text {stat }} \pm 0.78_{\text {sys }}\right) \times 10^{-11} \mathrm{~cm}^{-2} \mathrm{~s}^{-1}$ above $0.2 \mathrm{TeV}$ during the period of the VERITAS observations. The source is strongly variable on a daily timescale across all wavebands, from optical to $\mathrm{TeV}$, with a peak flux corresponding to $\sim 0.3$ times the steady Crab Nebula flux at $\mathrm{TeV}$ energies. Follow-up observations in the optical and $\mathrm{X}$-ray bands classify the newly discovered $\mathrm{TeV}$ source as
\end{abstract}


a BL Lac-type blazar with uncertain redshift, although recent measurements suggest $z=0.108$. VER J0521+211 exhibits all the defining properties of blazars in radio, optical, X-ray, and gamma-ray wavelengths.

Key words: BL Lacertae objects: individual (VER J0521+211) - gamma rays: galaxies

Online-only material: color figures

\section{INTRODUCTION}

$\mathrm{TeV}$ astronomy is concerned with the detection of astrophysical gamma rays with energies greater than $\sim 0.1 \mathrm{TeV}$. The most sensitive detectors in this energy region are the current generation of ground-based imaging atmospheric Cherenkov telescopes: VERITAS (Holder et al. 2008), H.E.S.S. (Hinton 2004), and MAGIC (Albert et al. 2008a), which can detect sources with a flux less than 0.01 times the steady flux of the Crab Nebula (hereafter "Crab"; Hillas et al. 1998) with an exposure of a few tens of hours. The angular resolution of current instruments is $\sim 0$. 1 , and the field of view is typically limited to a diameter of $<5^{\circ}$. Observations consist of surveying regions of the sky using many overlapping, noncontemporaneous exposures (Aharonian et al. 2006a; Weinstein 2009), or targeting locations of interest based on information provided by observations at other wavelengths.

The Large Area Telescope (LAT) on board the Fermi Gammaray Space Telescope is the first instrument to provide a view of the entire gamma-ray sky at energies that overlap with those accessible to ground-based telescopes. Figure 1 shows the arrival directions of all diffuse-class photon events ${ }^{40}$ recorded during the first year of Fermi-LAT observations with energies above $30 \mathrm{GeV}$. Clearly, the highest-energy Fermi-LAT observations provide an excellent guide to the TeV sky, and can be used to select additional $\mathrm{TeV}$ candidates for targeted observations. Numerous authors have addressed this, producing catalogs based on $>100 \mathrm{GeV}$ Fermi-LAT events (Neronov et al. 2010) and lists of $\mathrm{TeV}$ source candidates (e.g., Abdo et al. 2009). In this work, the presence of a cluster of high-energy photons (Figure 1) spatially associated with an X-ray source was used to motivate observations with the VERITAS array of a previously unobserved location, leading to the detection of a new bright TeV source: VER J0521+211. The only other cluster flagged by this method (1ES 0502+675) was already being observed by VERITAS at the time and was subsequently detected after $13 \mathrm{hr}$ of exposure (Ong 2009b).

This paper presents the discovery of gamma-ray emission from VER J0521+211, and a detailed study of its observational properties from radio frequencies to gamma-ray energies. The paper is structured as follows: Sections 2-5 present the observations and analysis results by VERITAS (TeV), Fermi-LAT $(\mathrm{GeV})$, Swift (X-ray), and MDM and Steward Observatory (optical), respectively. The identification of VER J0521+211 as a new TeV blazar is discussed in Section 6, and its main observational properties are presented in Section 7. An upper limit on the redshift of VER J0521+211 is calculated in Section 8, and the overall spectral energy distribution (SED) is discussed in Section 9. Finally, Section 10 summarizes the main conclusions of the study.

\footnotetext{
40 IRF version P6V3, see http://fermi.gsfc.nasa.gov/ssc/data/analysis/
} documentation/Cicerone/Cicerone_Data/LAT_DP.html.

\section{VERITAS OBSERVATIONS}

The VERITAS observatory is described in detail in Holder et al. (2008) and Holder et al. (2006). The array consists of four $12 \mathrm{~m}$ diameter imaging atmospheric Cherenkov telescopes, with photomultiplier (PMT) cameras covering a field of view of 3.5. The array has a total effective area of $\sim 5 \times 10^{4} \mathrm{~m}^{2}$ between 0.2 and $10 \mathrm{TeV}$. Following the relocation of the original prototype telescope to a more favorable position in Summer 2009 (Perkins et al. 2009), VERITAS has sensitivity to detect a source with $0.01 \mathrm{Crab}$ flux in under $30 \mathrm{hr}$ of observations. The angular and energy resolution for reconstructed gamma-ray showers is energy dependent, reaching $\sim 0.1$ and $15 \%$, respectively, at $1 \mathrm{TeV}$.

Inspection of the $>30 \mathrm{GeV} \mathrm{Fermi-LAT} \mathrm{map} \mathrm{(Figure} \mathrm{1)} \mathrm{led} \mathrm{to}$ the identification of a cluster of high-energy photons, which was used to trigger VERITAS observations centered around R.A. = $05^{\mathrm{h}} 21^{\mathrm{m}} 46^{\mathrm{s}}$, decl. $=+21^{\circ} 12^{\prime} 51^{\prime \prime} .5(\mathrm{~J} 2000)$, corresponding to the position of RGB J0521.8+2112, the only radio/X-ray source within 0.1 of the LAT excess.

Observations took place between MJD 55126 (2009 October 22) and MJD 55212 (2010 January 16) and consisted of 20 minute exposures in wobble observing mode (Fomin et al. 1994), taken at a mean zenith angle of $16^{\circ}$. After excluding data taken under poor weather conditions or with hardware problems, the data set comprises $14.5 \mathrm{hr}$ of dead-time-corrected exposure.

VERITAS data analysis follows the procedure outlined in Acciari et al. (2008). Cherenkov light from air showers initiated by gamma rays and cosmic rays triggers the readout of PMT signals, which are then calibrated and used to reconstruct an image of the shower in the focal plane. Individual telescope images are parameterized by simple moment analysis (Hillas 1985), and geometrical reconstruction is used to calculate the arrival direction of the primary, $\theta$, defined with respect to the position of the candidate source location on the sky. The image shape in each telescope is compared with the expected shapes for gamma-ray showers generated using Monte Carlo simulations and the differences, averaged over all telescopes, are used to derive the mean-reduced-scaled width and meanreduced-scaled length parameters, as defined in Acciari et al. (2008). Gamma-ray-like events are selected by applying cuts on the various image parameters. The cuts used in this work are mean-reduced-scaled width/length between -1.2 and 0.5 , $\theta<0$. 1 , and at least three telescope images with an integrated signal size per image equivalent to $>94$ photoelectrons. These cuts are designed to provide optimum sensitivity to a moderately strong point-like gamma-ray source $(0.05 \mathrm{Crab})$ with a Crab-like differential power-law photon index of $\sim 2.5$. Over $99.9 \%$ of the background cosmic ray events are removed by these cuts. The number of background events remaining in the signal region is estimated from 10 off-source regions within the same field of view using the reflected region technique (Aharonian et al. 2001; Berge et al. 2007).

After data selection, reduction, and signal extraction, an excess of 221 candidate gamma-ray events over a background of 119 is detected at the location of RGB J0521.8+2112, 


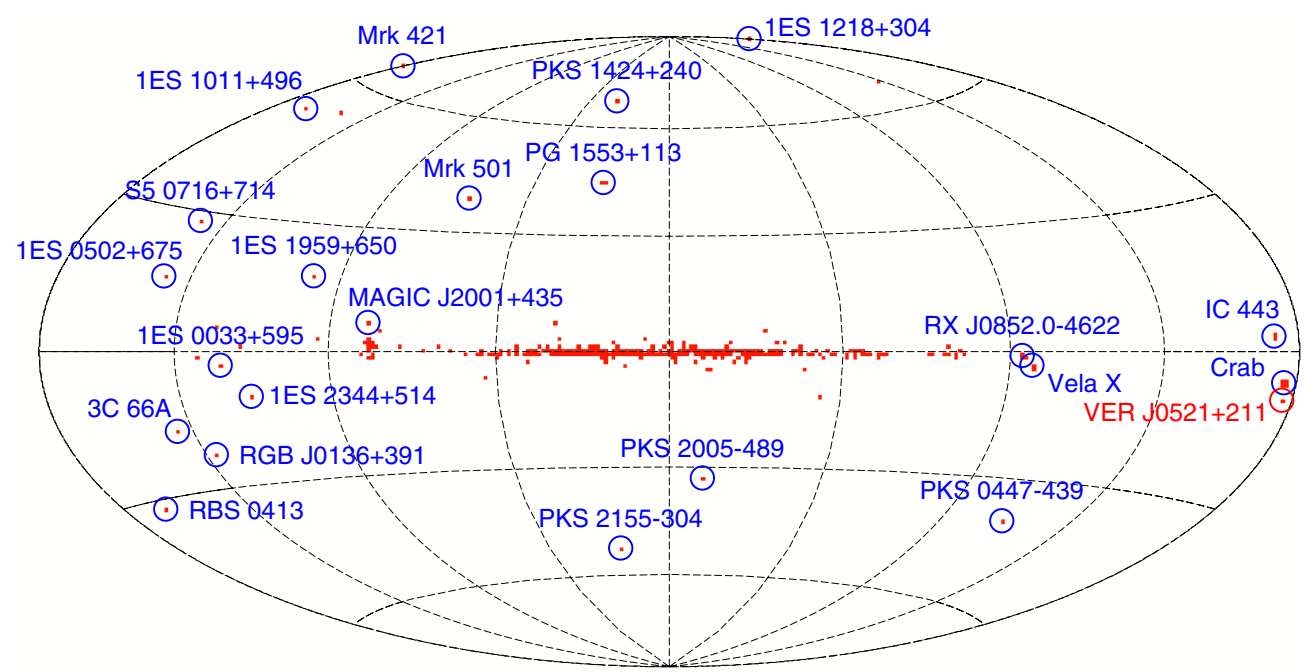

Figure 1. Map, in Galactic coordinates, showing the arrival directions of all diffuse-class photon events ${ }^{41}$ with zenith angle $<105^{\circ}$ recorded by Fermi-LAT between 2008 August 4 and 2009 August 25 with reconstructed energies greater than $30 \mathrm{GeV}$. The events are binned in $1^{\circ}$ bins, and bins containing at least five events are shaded red. Known $\mathrm{TeV}$ sources coincident with shaded bins are indicated in regions where source confusion or diffuse background is not an issue.

(A color version of this figure is available in the online journal.)

corresponding to a significance of 15.6 standard deviations according to Equation (17) in Li \& $\mathrm{Ma}$ (1983, with $N_{\text {on }}=340$, $N_{\text {off }}=1518$, and $\left.\alpha=0.08\right)$. The angular distribution of the excess events is compatible to that of a point-like source given the instrumental point spread function of $6^{\prime}$ for $68 \%$ containment radius. The position of the source is derived by fitting a symmetric two-dimensional Gaussian to the uncorrelated excess map, resulting in a best-fit centroid at R.A. = $05^{\mathrm{h}} 21^{\mathrm{m}} 45^{\mathrm{s}}$, decl. $=+21^{\circ} 12^{\prime} 51^{\prime \prime} .4$, with a statistical uncertainty of $14^{\prime \prime}$ and a systematic uncertainty of $25^{\prime \prime}$, dominated by the telescopes' pointing accuracy. The new $\mathrm{TeV}$ source is cataloged as VER J0521+211, based on the name first reported in Ong (2009a).

Figure 2 shows the time-averaged energy spectrum of VER J0521+211, extending from a threshold energy of 0.2 to $\sim 1 \mathrm{TeV}$. It is well described by a power law $d N / d E=N_{0}(E / 0.4 \mathrm{TeV})^{-\Gamma}$ with normalization $N_{0}=$ $\left(1.99 \pm 0.18_{\text {stat }} \pm 0.80_{\text {syst }}\right) \times 10^{-11} \mathrm{~cm}^{-2} \mathrm{~s}^{-1} \mathrm{TeV}^{-1}$ and photon index $\Gamma=3.44 \pm 0.20_{\text {stat }} \pm 0.30_{\text {syst }}$. The time-averaged integral photon flux is $F_{>0.2 \mathrm{TeV}}=\left(1.93 \pm 0.13_{\text {stat }} \pm 0.78_{\text {syst }}\right) \times$ $10^{-11} \mathrm{~cm}^{-2} \mathrm{~s}^{-1}$, corresponding to $0.092 \pm 0.006 \mathrm{Crab}(1 \mathrm{Crab}=$ $2.1 \times 10^{-10} \mathrm{~cm}^{-2} \mathrm{~s}^{-1}$; Hillas et al. 1998). Compatible results were obtained using two independent analysis packages.

Flux variability was explored by producing a 1 day binned light curve during the VERITAS observations (Figure 3, top). After a first detection in 2009 October 22-24 (Ong 2009a) with a derived $\mathrm{TeV}$ flux of $0.09 \pm 0.01 \mathrm{Crab}$, a later round of observations revealed a higher flux level, peaking at $0.33 \pm 0.07 \mathrm{Crab}$ on 2009 November 27 (MJD 55162, hereafter "TeV flare"). A $\chi^{2}$ fit to the nightly flux points for constant emission gives a probability of $7 \times 10^{-7}$, indicating flux variability at a confidence level of 5.8 standard deviations.

Spectral variability was tested by deriving a "low-state" spectrum from the data taken during the first period of VERITAS observations (2009 October 22-30, MJD 55126 - 55134), and a "flare" spectrum from the night of 2009 November 27. A power-law fit to each spectrum yields $\Gamma_{\text {low }}=2.92 \pm 0.34$ and $\Gamma_{\text {flare }}=3.25 \pm 0.72$. The difference in the reconstructed photon index does not constitute significant evidence for spectral variability in the $\mathrm{TeV}$ band.

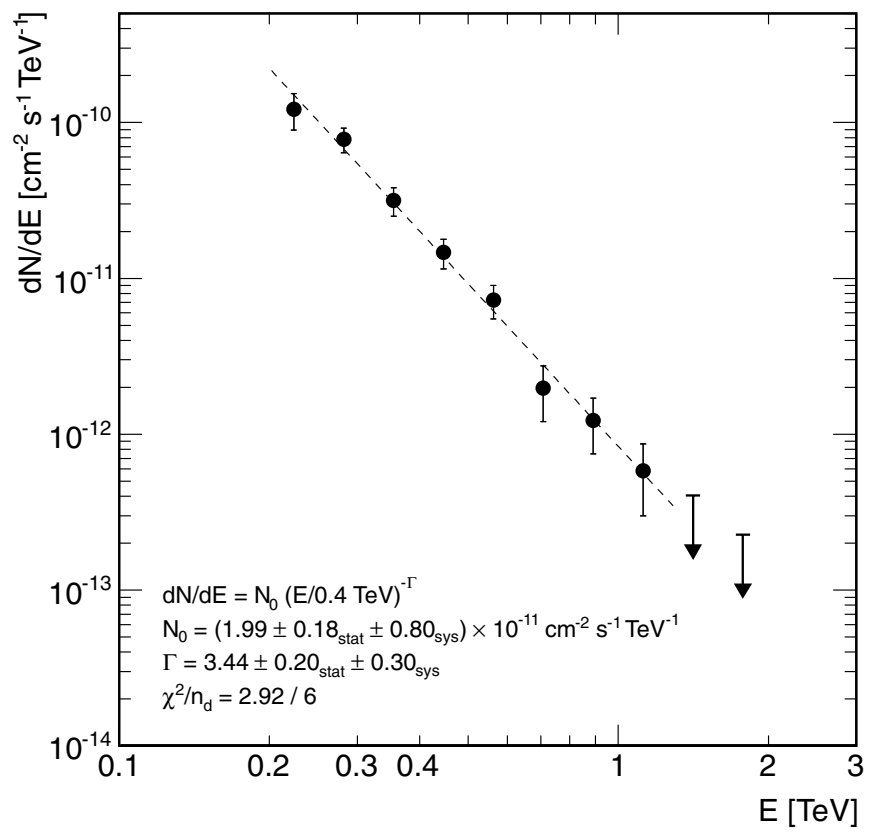

Figure 2. Time-averaged differential photon spectrum of VER J0521+211, obtained during VERITAS observations between MJD 55126 (2009 October 22) and MJD 55212 (2010 January 16). Upper limits at 95\% confidence level are shown for the two highest energy points, where the significance is $<2 \sigma$.

\section{FERMI-LAT OBSERVATIONS}

Fermi-LAT is a pair-conversion telescope sensitive to gamma rays in the range from 0.02 to more than $300 \mathrm{GeV}$ with a field of view of $\sim 2.4 \mathrm{sr}$. The effective area of the LAT changes with energy and incidence angle, being $\sim 0.8 \mathrm{~m}^{2}$ for on-axis photons with $E>10 \mathrm{GeV}$. Full details about the instrument and its performance are given in Atwood et al. (2009) and Ackermann et al. (2012).

A GeV source spatially associated with VER J0521+211 was listed in the LAT 11 month catalog (1FGL J0521.7+2114; Abdo et al. 2010a) and confirmed in the second source catalog (2FGL J0521.7+2113; Nolan et al. 2012) and in the catalog of sources detected above $10 \mathrm{GeV}$ (1FHL J0521.7+2113; Ackermann et al. 


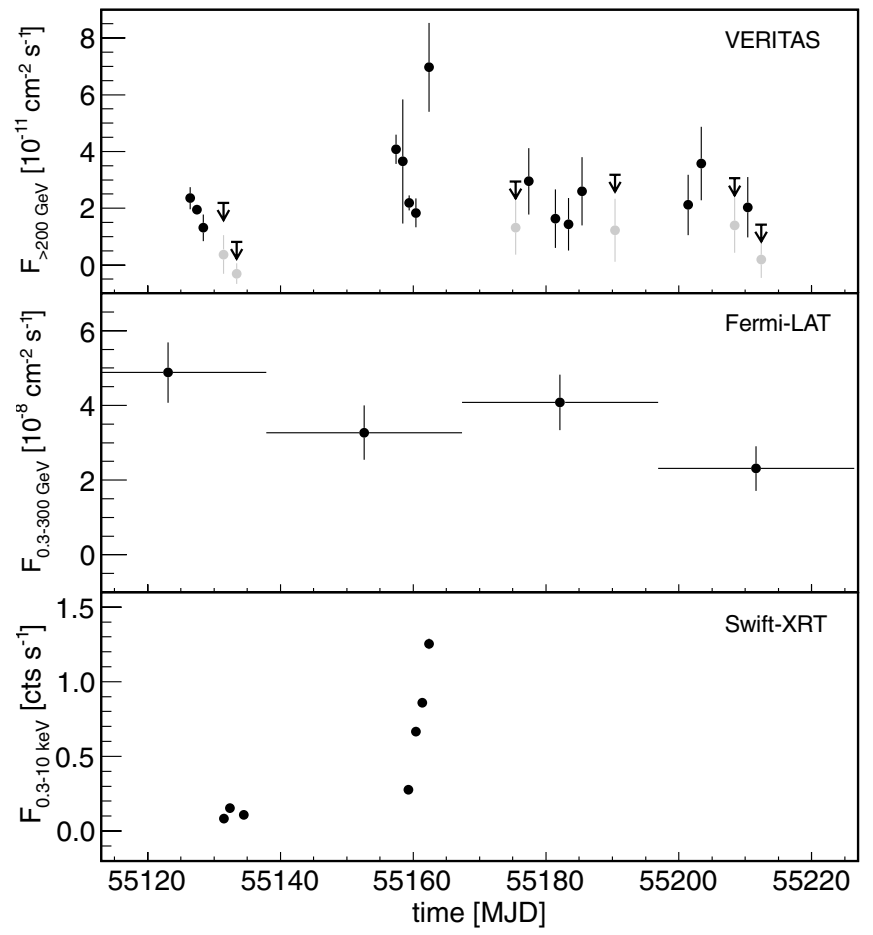

Figure 3. VERITAS $(E>0.2 \mathrm{TeV})$, Fermi-LAT $(0.1-300 \mathrm{GeV})$, and Swift/ XRT (0.3-10 keV) light curves of VER J0521+211. Photon fluxes are calculated in 1 day bins for VERITAS and Swift/XRT, and 29.5 days for Fermi-LAT. The VERITAS light curve shows significant flux points (black dots) when the signal exceeds $2 \sigma$, and $95 \%$ confidence level upper limits (black arrows) together with flux points (gray dots) for marginal detections. The error bars on the Swift/XRT rates are at the $\sim 2 \%-7 \%$ level, and are not visible in the plot.

2013). The region was analyzed in more detail by selecting source-class ${ }^{41}$ events with reconstructed energy $0.3<E<$ $300 \mathrm{GeV}$ collected during the period of VERITAS observations (MJD 55126 - 55212) in a $20^{\circ} \times 20^{\circ}$ region of interest (RoI) centered at the location of VER J0521+211. Spectral parameters were extracted by fitting a model containing the Galactic and isotropic diffuse background ${ }^{42}$ and point sources from the $2 \mathrm{FGL}$ catalog. The analysis was done using binned likelihood as implemented in the Fermi-LAT Science Tools v09-26-00 with P7V6 response functions and the standard quality selection cuts described in Nolan et al. (2012). The energy spectrum (light curve) were derived following the procedure detailed in Nolan et al. (2012), dividing the data into bins of energy (time) and performing a likelihood analysis with the spectral shape parameters frozen for all sources in the model.

During the period of VERITAS observations, VER $\mathrm{J} 0521+211$ was detected by the LAT with a test statistic of $237(\sim 15 \sigma)$. The derived integral photon flux is $F_{0.3}-300 \mathrm{GeV}=$ $(2.8 \pm 0.4) \times 10^{-8} \mathrm{~cm}^{-2} \mathrm{~s}^{-1}$, with a spectrum well described by a power law with photon index $\Gamma=1.72 \pm 0.09$. An identical analysis of an extended data set collected by Fermi ( $\sim 46$ months) reveals that VER J0521+211 was in a harder spectral state during the VERITAS observations, compared to a time-averaged photon index of $\Gamma=1.97 \pm 0.03$, while the flux level was comparable over both periods.

\footnotetext{
41 See http://fermi.gsfc.nasa.gov/ssc/data/analysis/documentation/ Cicerone/Cicerone_Data/LAT_DP.html.

42 gal_2yearp7v6_v0.fits and iso_p7v6source.txt, respectively.
}

Variability was tested by extracting the source flux in 29.5 day bins. ${ }^{43}$ Applying a likelihood-based variability test (Nolan et al. 2012) to the 46 months of data gives a probability of a constant flux of $2 \times 10^{-20}$, indicating flux variability in the $\mathrm{GeV}$ band at a confidence level of 9.3 standard deviations. The 46 month light curve (not shown) exhibits flux changes by a factor of $\sim 4$ between the lowest and highest emission levels.

An additional test for variability above $1 \mathrm{GeV}$ was performed using a Bayesian Block method (Scargle 1998). For this test, source-class events with $E>1 \mathrm{GeV}$ were extracted from a $1^{\circ}$ radius RoI centered on the source coordinates. The data were divided into time blocks over which the event rate was compatible with a constant value. The optimal width of the blocks was determined by maximum likelihood analysis using the algorithm described in Jackson et al. (2005). The effective exposure associated to each event is taken into account, correcting for exposure variations caused by the motion of the spacecraft. A $1 \%$ false-positive threshold was used for detecting variability. The method identifies three periods of different constant flux with durations between 310 and 451 days, indicating variability above $1 \mathrm{GeV}$. The highest flux state, with boundaries MJD 54993-55419, includes the VERITAS observations. However, no significant evidence for shorter time variability is found in the Fermi-LAT data above $1 \mathrm{GeV}$ during VERITAS observations, or in coincidence with the X-ray and TeV flare on MJD 55162.

\section{SWIFT OBSERVATIONS}

After the discovery of $\mathrm{TeV}$ emission by VERITAS (Ong 2009a), Swift X-Ray Telescope (XRT; Gehrels et al. 2004) observations were triggered. Seven exposures were obtained between 2009 October 27 and November 27. The total observation time is $16.6 \mathrm{ks}$ distributed in exposures of $\sim 2.5 \mathrm{ks}$.

Swift/XRT data were analyzed with HEAsoft 6.9 and XSPEC 12.6.0 using the most recent calibration files as described in Burrows et al. (2005). All data were taken in photon counting mode. Pile-up effects were accounted for by extracting the signal from an annular source region when rates exceeded 0.5 counts $\mathrm{s}^{-1}$.

The Swift/XRT field of view of 23'.6 $\times 23$ ' 6 completely covers the VERITAS error circle. A single X-ray source was detected at R.A. $=05^{\mathrm{h}} 21^{\mathrm{m}} 45^{\mathrm{s}} .98$, decl. $=+21^{\circ} 12^{\prime} 52^{\prime \prime} .9$ (with $1^{\prime \prime} .9$ location uncertainty), spatially coincident with RGB J0521.8+2112, as shown in Figure 4. The measured, pile-up-corrected count rate is significantly variable on a daily timescale (Figure 3), with a flux increase up to a factor of $\sim 15$ between low and high states. For spectral reconstruction, each observation was binned and fit with an absorbed power law, with neutral hydrogen column density taken from Kalberla et al. (2005). The three exposures from 2009 October 27-30 showed a lower source count rate $\left(\sim 0.1\right.$ counts $\left.s^{-1}\right)$ and were combined for the spectral analysis. The derived photon index continuously hardens from $2.47 \pm 0.10$ when the emission is lowest to $2.00 \pm 0.04$ on the night of the highest X-ray flux (MJD 55162), when RGB J0521.8+2112 reaches a peak flux of $(3.14 \pm 0.14) \times 10^{-11} \mathrm{erg} \mathrm{cm}^{-2} \mathrm{~s}^{-1}$ in the $2-10 \mathrm{keV}$ band.

\footnotetext{
43 The length of the bins is equal to the lunar period. Since VERITAS does not observe during full moon, this ensures that each epoch of VERITAS observations falls within one bin of the LAT light curve.
} 


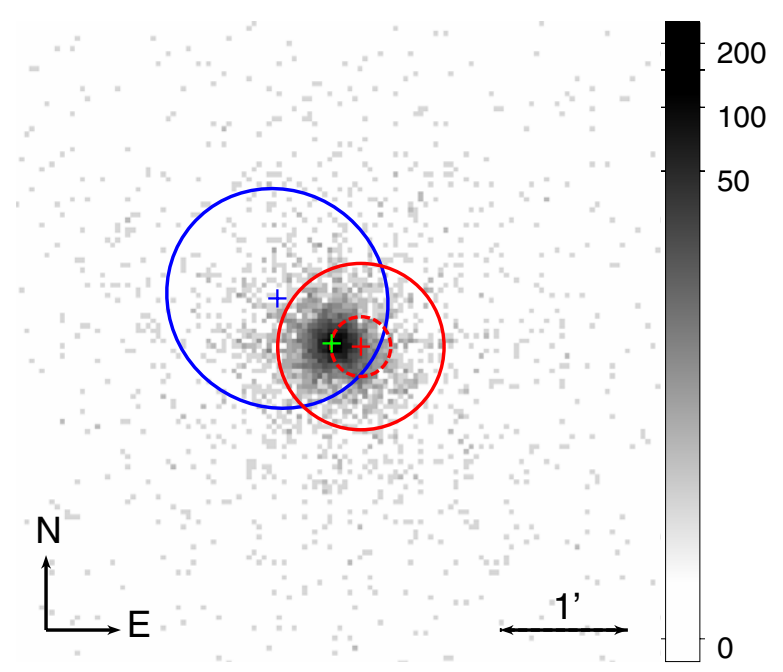

Figure 4. Swift/XRT cumulative counts map in the $0.3-10 \mathrm{keV}$ band, zoomed-in at the location of VER J0521+211. The field is $5^{\prime} \times 5^{\prime}$, with gray code indicating counts on a logarithmic scale. The green cross indicates the best-fit centroid to the Swift/XRT data. The red cross shows the position of VER J0521+211 with red dashed circle indicating the VERITAS 68\% statistical location error and red-solid line showing statistical and systematic error. The blue ellipse displays $68 \%$ error contour of 2FGL J0521.7+2113 as measured by Fermi-LAT (Nolan et al. 2012).

(A color version of this figure is available in the online journal.)

\section{OPTICAL OBSERVATIONS}

Beginning with the radio-interferometric position from Beasley et al. (2002), the optical counterpart of RGB J0521.8+2112 was identified on the digitized sky survey, with coordinates R.A. $=05^{\mathrm{h}} 21^{\mathrm{m}} 45^{\mathrm{s}} .96$, decl. $=+21^{\circ} 12^{\prime} 51^{\prime \prime} \cdot 6$, and magnitudes $B 2=17.32, R 2=15.47$, and $I 2=15.06$ listed in the USNO B1.0 catalog (Monet et al. 2003). Optical spectroscopic observations were made on three occasions at the $2.4 \mathrm{~m}$ Hiltner telescope of the MDM Observatory, using three different CCD spectrographs. The optical spectra (Figure 5) are devoid of intrinsic emission or absorption lines and stellar continuum emission in the wavelength range 4000-7500 , classifying the source as a BL Lac-type blazar but not revealing its redshift. Features marked in Figure 5 are absorption lines from the Galactic interstellar medium at zero redshift. Recently published observations with the Low Resolution Imaging Spectrograph at the W. M. Keck Observatory (Shaw et al. 2013) show a weak emission feature identified as $[\mathrm{N}$ II] $\lambda \lambda 6548,6583$, which would indicate a redshift of $z=0.108$. Although this feature cannot be identified in the MDM spectra, the measurements are not in conflict given the lower level of continuum emission present in the spectrum by Shaw et al. (2013) and the higher sensitivity of Keck. The first spectrum of RGB J0521.8+2112 in Figure 5 was obtained on 2009 October 27, only five days after the first VERITAS observations. The calibrated flux in this spectrum corresponds to $B \sim 17.0, R \sim 15.2$, slightly brighter than the USNO B1.0 magnitudes. It appears that the optical flux faded
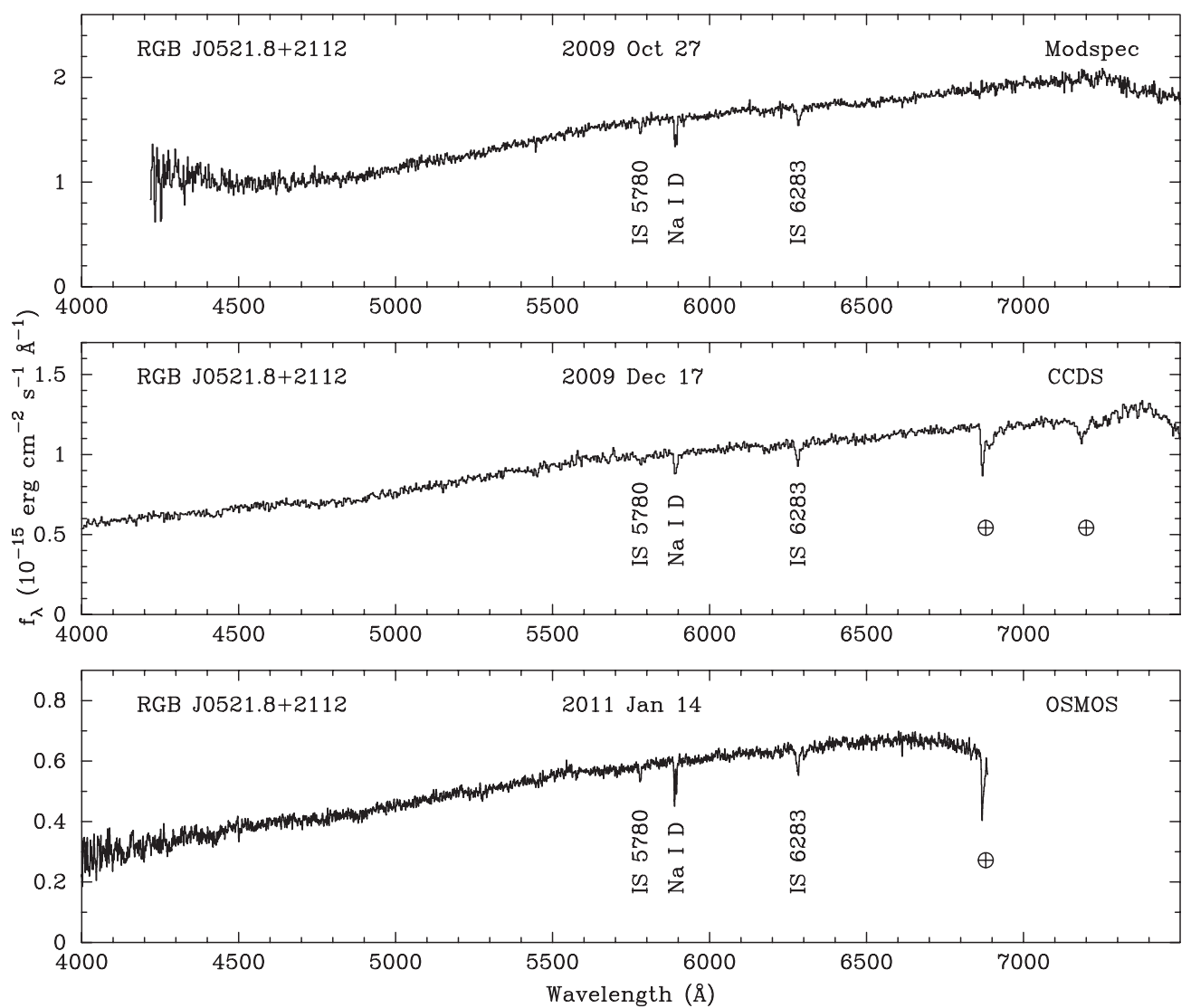

Figure 5. Optical spectra of RGB J0521.8+2112 obtained on the $2.4 \mathrm{~m}$ Hiltner telescope of the MDM Observatory. The Modspec spectrum was obtained with an exposure of $2 \times 600 \mathrm{~s}$ and a resolution of $4 \AA$. The exposures for the CCDS and OSMOS spectra were $3 \times 1800 \mathrm{~s}$ with resolution of $8 \AA$ and $3 \AA$, respectively. Galactic interstellar absorption lines are labeled with their wavelength, including the Na I D doublet and diffuse interstellar bands at $5780 \AA$ and $6283 \AA$. These are relatively prominent because of the low Galactic latitude of -8.7 and the corresponding visual extinction $A_{V}=1.9$ (Schlafly \& Finkbeiner 2011) to the source. Telluric absorption by $\mathrm{O}_{2}$ and $\mathrm{H}_{2} \mathrm{O}$ are indicated in the CCDS and OSMOS spectra; in the case of Modspec they were corrected using the spectrum of a featureless B star. 
Table 1

Optical Polarimetry of RGB J0521.8+2112

\begin{tabular}{llcr}
\hline \hline Object & Date & $\begin{array}{c}P \\
(\%)\end{array}$ & $\begin{array}{c}\theta \\
\left({ }^{\circ}\right)\end{array}$ \\
\hline RGB J0521.8+2112 & 2011 Nov 24 & $3.24 \pm 0.13$ & $13.6 \pm 1.1$ \\
RGB J0521.8+2112 & 2011 Nov 28 & $4.47 \pm 0.14$ & $12.7 \pm 0.9$ \\
RGB J0521.8+2112 & 2011 Dec 1 & $6.50 \pm 0.14$ & $22.0 \pm 0.6$ \\
\hline Reference star A & 2011 Nov 28 & $1.66 \pm 0.07$ & $146.7 \pm 1.2$ \\
Reference star B & 2011 Nov 28 & $0.69 \pm 0.04$ & $148.9 \pm 1.7$ \\
\hline ISP corrected values & & & \\
RGB J0521.8+2112 & 2011 Nov 24 & $3.74 \pm 0.15$ & $26.8 \pm 1.1$ \\
RGB J0521.8+2112 & 2011 Nov 28 & $4.83 \pm 0.16$ & $22.7 \pm 0.9$ \\
RGB J0521.8+2112 & 2011 Dec 1 & $7.26 \pm 0.16$ & $28.2 \pm 0.6$ \\
\hline
\end{tabular}

in the subsequent months and years by $\sim 1 \mathrm{mag}$, although none of the spectrophotometry is precise because of the narrow spectrograph slit.

Optical linear polarimetry was performed using the Steward Observatory $1.54 \mathrm{~m}$ Kuiper Telescope, located on Mt. Bigelow, AZ. RGB J0521.8+2112 was observed on 2011 November 24, 28, and December 1 with the SPOL CCD imaging/ spectropolarimeter (Schmidt et al. 1992). High signal-to-noise broadband measurements were derived by binning the polarization spectra in the range of 5000-7000 $\AA$. All measurements are summarized in Table 1 and have been corrected for statistical bias (Wardle \& Kronberg 1974). Given the low Galactic latitude of the source, two field stars were also observed, suggesting a significant interstellar polarization (ISP) along the sight line to RGB J0521.8+2112 (see Table 1). The binned spectropolarimetry of RGB J0521.8+2112, corrected for an estimate of the ISP, yields a variable degree of polarization $(P)$ between $P=3.74 \% \pm 0.15 \%$ with position angle $\theta=26.8 \pm 1.1$ (November 24) and $P=7.26 \% \pm 0.16 \%$ at $\theta=28.2 \pm 0.6$ (December 1). Because of the variability in $P$ observed for RGB J0521.8+2112, the object must be intrinsically polarized regardless of the actual level of ISP in this line of sight.

\section{IDENTIFICATION OF THE GAMMA-RAY SOURCE}

The observations that led to the discovery of VER J0521+211 were triggered by the identification of a cluster of photons with $E>30 \mathrm{GeV}$ in the first year of Fermi-LAT data (Figure 1). The most likely counterpart of the LAT excess was RGB J0521.8+2112, which had no classification, although sources in the ROSAT/Green Bank catalog are generally associated with active galactic nuclei (AGNs; Laurent-Muehleisen et al. 1997). RGB J0521.8+2112 is the only radio source spatially compatible with the LAT excess in a high-completeness very long baseline interferometry sample down to $0.15 \mathrm{Jy}$, indicative of a radio-loud blazar (see Kovalev 2009). No other spatially associated X-ray, UV, or compact radio source was found in archival catalogs.

Immediately after the VERITAS detection, X-ray and optical observations were triggered to confirm the extragalactic nature of VER J0521+211. Swift/XRT establishes RGB J0521.8+2112 as the only bright X-ray source inside the 39" VERITAS error circle (Figure 4). The identification of VER J0521+211 with RGB J0521.8+2112 is further supported by the detection of a $\mathrm{TeV}$ flare simultaneously seen in X-rays (Figure 3). However, the statistical significance of the overall X-ray/TeV flux correlation cannot be assessed due to the limited number of flux measurements in both bands.
Table 2

Observational Properties of VER J0521+211 during VERITAS Observations

\begin{tabular}{lcc}
\hline \hline & Low State & Flare \\
\hline Radio spectral index $\left(\alpha_{\mathrm{r}}\right)^{\mathrm{a}}$ & \multicolumn{2}{c}{$0.16 \pm 0.01$} \\
Optical polarization $(P)$ & $0.16 \pm 0.2$ & $3.7 \%-7.3 \%$ \\
X-ray flux $\left(F_{2-10 \mathrm{keV}}\right)^{\mathrm{b}}$ & $2.5 \pm 0.1$ & $2.0 \pm 0.1$ \\
X-ray photon index $\left(\Gamma_{\mathrm{x}}\right)^{\mathrm{c}}$ & \multicolumn{2}{c}{$2.8 \pm 0.4$} \\
GeV photon flux $\left(F_{0.3-300 \mathrm{GeV}}\right)^{\mathrm{d}}$ & \multicolumn{2}{c}{$1.7 \pm 0.1$} \\
GeV photon index $\left(\Gamma_{\mathrm{GeV}}\right)$ & $0.09 \pm 0.01$ & $0.33 \pm 0.07$ \\
TeV photon flux $\left(F_{>0.2 \mathrm{TeV}}\right)^{\mathrm{e}}$ & $2.9 \pm 0.3$ & $3.3 \pm 0.7$ \\
TeV photon index $\left(\Gamma_{\mathrm{TeV}}\right)$ & $2.4 \times 10^{44}$ & $8.8 \times 10^{44}$ \\
TeV luminosity $\left(L_{>0.2 \mathrm{TeV}}\right)^{\mathrm{f}}$ & \multicolumn{2}{c}{69} \\
Radio loudness $\left(R_{\mathrm{rB}}\right)^{\mathrm{g}}$ & 0.42 & 0.46 \\
Radio-to-optical slope $\left(\alpha_{\mathrm{ro}}\right)^{\mathrm{h}}$ & 1.31 & 0.77 \\
Optical-to-X-ray slope $\left(\alpha_{\mathrm{ox}}\right)^{\mathrm{i}}$ & 1.8 & 0.8 \\
Compton dominance $\left(R_{C}\right)^{\mathrm{j}}$ & \multicolumn{2}{c}{$0.108^{\mathrm{k}}(<0.34)^{\mathrm{l}}$} \\
Redshift $(z)$ & \multicolumn{3}{c}{} \\
\hline
\end{tabular}

Notes.

${ }^{\mathrm{a}} F_{v} \propto v^{-\alpha_{\mathrm{r}}}$.

b $10^{-11} \mathrm{erg} \mathrm{cm}^{-2} \mathrm{~s}^{-1}$.

c $d N / d E \propto E^{-\Gamma}$.

d $10^{-8} \mathrm{~cm}^{-2} \mathrm{~s}^{-1}$.

${ }^{\text {e }} \mathrm{Crab}\left(2.1 \times 10^{-10} \mathrm{~cm}^{-2} \mathrm{~s}^{-1}\right.$; Hillas et al. 1998).

f $\operatorname{erg~s}^{-1}$.

g $R_{\mathrm{rB}}=L_{5 \mathrm{GHz}} / L_{B_{\mathrm{mag}}}$.

${ }^{\mathrm{h}} \alpha_{\mathrm{ro}}=-\log \left(F_{1.4 \mathrm{GHz}} / F_{R_{\mathrm{mag}}}\right) / \log \left(v_{1.4 \mathrm{GHz}} / \nu_{R_{\mathrm{mag}}}\right)$.

${ }^{\mathrm{i}} \alpha_{\mathrm{ox}}=-\log \left(F_{R_{\mathrm{mag}}} / F_{1 \mathrm{keV}}\right) / \log \left(v_{R_{\mathrm{mag}}} / \nu_{1 \mathrm{keV}}\right)$.

${ }^{\mathrm{j}} R_{C}=L_{\mathrm{HE}} / L_{\mathrm{sy}}$.

k Shaw et al. (2013).

${ }^{1}$ Derived in Section 8.

The flux variability observed in the TeV band further suggests that VER J0521+211 could indeed be an AGN. To date, all known variable $\mathrm{TeV}$ sources are AGNs, with the exception of the four detected gamma-ray binaries (LSI +61 303, HESS J0632+057, PSR B1259-63, and LS 5039) and the Crab pulsar, which is variable at a much shorter timescale $(33 \mathrm{~ms})$.

The optical counterpart of VER J0521+211 was subsequently identified through observations at MDM. Optical spectroscopy revealed a continuum-dominated spectrum (Figure 5), unambiguously identifying VER J0521+211 as a BL Lac-type blazar.

\section{OBSERVATIONAL PROPERTIES OF VER J0521+211}

The main observational properties of VER J0521+211 are summarized in Table 2. Taking the $5 \mathrm{GHz}$ flux from White $\&$ Becker (1992) and the $B$-band apparent magnitude from MDM observations, the radio-to-optical luminosity ratio for VER J0521+211 is $R_{\mathrm{rB}}=L_{5 \mathrm{GHz}} / L_{B_{\mathrm{mag}}}=69$, implying a radioloud AGN ( $R_{\mathrm{rB}}>10$; Kellermann et al. 1989) with prominent radio jet or lobe emission (Urry \& Padovani 1995). The spectrum of the unresolved core emission of VER J0521+211 in the radio band is well described by a power law, with $F_{v} \propto v^{-\alpha_{\mathrm{r}}}$ and spectral index $\alpha_{\mathrm{r}}=0.16 \pm 0.01$ (Figure 6), compatible with $\alpha_{\mathrm{r}} \leqslant 0.5$, characteristic of jet-dominated flat-spectrum radio sources.

The radio jet of RGB J0521.8+2112 has been imaged on milliarcsecond scales in five epochs between 2009 October and 2012 April with the Very Long Baseline Array (VLBA) at $15 \mathrm{GHz}$ as part of the MOJAVE program (Lister et al. 2009a). The radio morphology consists of a bright radio core and an apparent one-sided jet that extends for $\sim 20$ mas to the west while curving slightly to the northwest (Figure 7). The radio core is very compact, with a brightness temperature above $10^{11} \mathrm{~K}$ at 


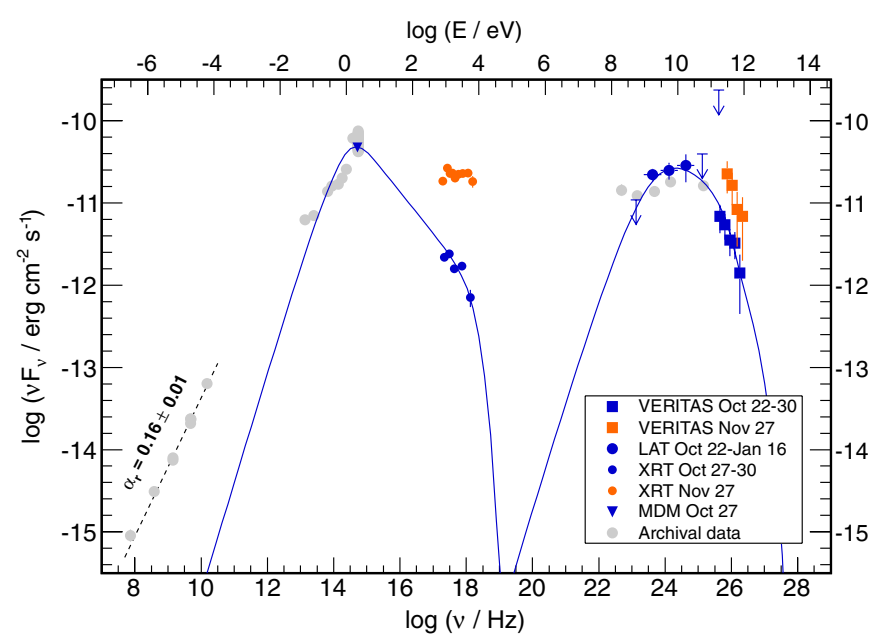

Figure 6. Spectral energy distribution of VER J0521+211 during the VERITAS detection. Optical, $\mathrm{X}$-ray, $\mathrm{GeV}$, and $\mathrm{TeV}$ data are shown for the low emission state (blue markers) and for the X-ray and TeV flare on 2009 November 27 (orange markers). Archival data points are shown in gray: radio (Cohen et al. 2007; Gregory et al. 1996; White \& Becker 1992; Jackson et al. 2007; Condon et al. 1998), infrared (Wright et al. 2010; Cutri et al. 2003), optical (Monet et al. 2003; Drake et al. 2009), and gamma rays (Nolan et al. 2012). Archival radio data at $15 \mathrm{GHz}$ is from the OVRO program (http://www.astro.caltech.edu/ovroblazars) and was obtained following Richards et al. (2011). Optical and infrared data are corrected for Galactic extinction using Schlafly \& Finkbeiner (2011). A dashed black line shows the fit of the radio data to a power law with $F_{v} \propto v^{-\alpha}$. The solid blue curve represents a one-zone SSC emission model with parameters adjusted to describe the low-state data, assuming $z=0.1$. Radio data points are not reproduced by the model, as they are expected to sample outer regions of the blazar jet, where the emission becomes optically thin to radio waves.

(A color version of this figure is available in the online journal.)

all epochs according to Gaussian model fits. The model fits to individual features in the jet do not reveal significant proper motions over the $2.5 \mathrm{yr}$ observation interval. During this time, however, there were significant changes in the polarization of the core and the inner 1.5 mas of the jet. The core remained weakly linearly polarized $(<1 \%)$, but increased steadily in polarized flux density from 0.7 to $1.6 \mathrm{mJy}$. The downstream jet polarization was typically much higher (up to $25 \%$ ) with electric polarization vectors perpendicular to the jet ridgeline, consistent with optically thin synchrotron emission associated with a relatively well-ordered, longitudinal jet magnetic field. A bright feature $\sim 1$ mas downstream of the core briefly flared in total intensity and polarization sometime between 2010 March and 2010 October, but returned to its earlier level by 2011 July. These short-timescale changes, as well as the one-sided jet morphology, high core compactness and jet polarization are very similar to those seen in highly Doppler-boosted blazar jets in the MOJAVE sample (Lister \& Homan 2005; Lister et al. 2009b).

Infrared observations of VER J0521+211 in the WISE Source Catalog ${ }^{44}$ show magnitudes of 10.6, 9.8, 7.6, and 5.6 in the 3.4, $4.6,12$, and $22 \mu \mathrm{m}$ bands, respectively. Its infrared colors are similar to those of known gamma-ray blazars (Massaro et al. 2012 b), providing further support for its identification as a $\mathrm{TeV}$ blazar (Massaro et al. 2012a).

The optical polarimetry reveals a highly polarized object that shows significant variations in both the level and position angle of polarization on timescales shorter than a week. This is strong evidence that a good fraction of the optical continuum of RGB J0521.8+2112 is produced by synchrotron radiation from

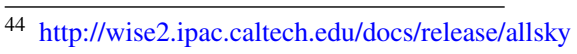

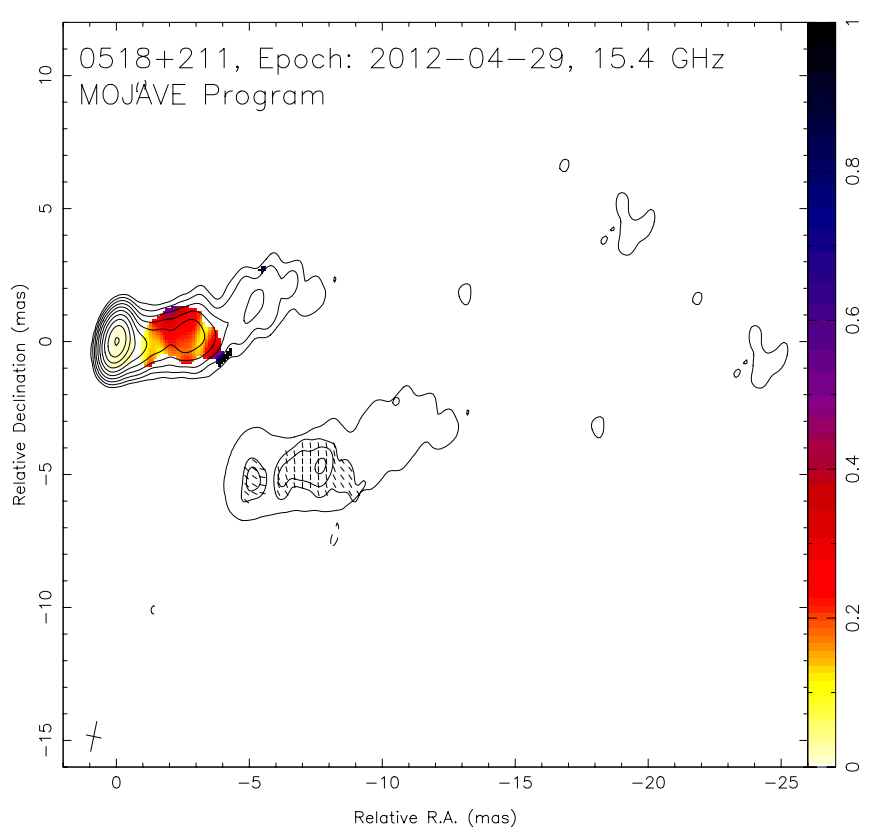

Figure 7. $15 \mathrm{GHz}$ MOJAVE VLBA composite image of RGB J0521.8+2112 on 2012 April 29. The top left image shows contours of total intensity, with fractional linear polarization overlaid in color scale. The bottom right image shows contours of linear polarization, plus a single outermost contour of total intensity. Electric polarization vector directions are plotted as equal-length ticks. The contour levels for total intensity are $0.5 \mathrm{mJy} \mathrm{beam}^{-1}$ in steps of 2 , while those for polarized intensity are for $0.7 \mathrm{mJy}$ beam $^{-1}$ in steps of 2 . The restoring beam is indicated in the bottom left corner, and has Gaussian FWHM dimensions 1.15 by 0.57 mas, with major axis at -11.4 from north.

(A color version of this figure is available in the online journal.)

regions with well-ordered magnetic fields. During the epoch of the Steward Observatory measurements, the position angle of the polarization $\left(20^{\circ}-30^{\circ}\right)$ was intermediate between being parallel and perpendicular to the axis of the radio jet.

BL Lac-type blazars are usually classified as low, intermediate, or high-frequency peaked BL Lacs according to the estimated peak frequency of their synchrotron component (LBL, $\log \left(v_{\text {sy }} / \mathrm{Hz}\right)<14$; IBL, $14-15$; HBL, > 15; e.g., Abdo et al. 2010b). Figure 8 shows the effective spectral indices between radio, optical, and X-ray bands for all known TeV blazars. Simultaneous flux measurements during "low-state" (2009 October 22-30) suggest that VER J0521+211 has synchrotron properties similar to those of the known IBLs. However, during the $\mathrm{X}$-ray and TeV flare, the brightening and hardening of the X-ray emission causes the synchrotron component of VER J0521+211 to show HBL-like properties (Figure 8). Spectral changes in the synchrotron component are not uncommon and have been reported in other TeV blazars (Massaro et al. 2008; Abdo et al. 2011c).

VER J0521+211 also shows similar luminosity in the synchrotron and high-energy peak of the SED (Figure 6), implying a Compton dominance $\left(R_{C}=L_{\mathrm{HE}} / L_{\mathrm{sy}}\right)$ in the range $0.8 \lesssim R_{C} \lesssim 1$.8. Such values are similar to those of high-power blazars (Meyer et al. 2012), which show synchrotron peak frequencies lower than "classical" HBLs $\left(\log \left(v_{\text {sy }} / \mathrm{Hz}\right) \lesssim 14.5\right.$; Meyer et al. 2011).

With a low-state TeV flux of $\sim 0.1 \mathrm{Crab}$ measured over three months of observations, and a flare of $\sim 0.3 \mathrm{Crab}, \mathrm{VER}$ $\mathrm{J} 0521+211$ is one of the brightest TeV blazars newly discovered by the current generation of Cherenkov telescopes. Other blazars, such as 3C 279 (Albert et al. 2008b), W Comae 


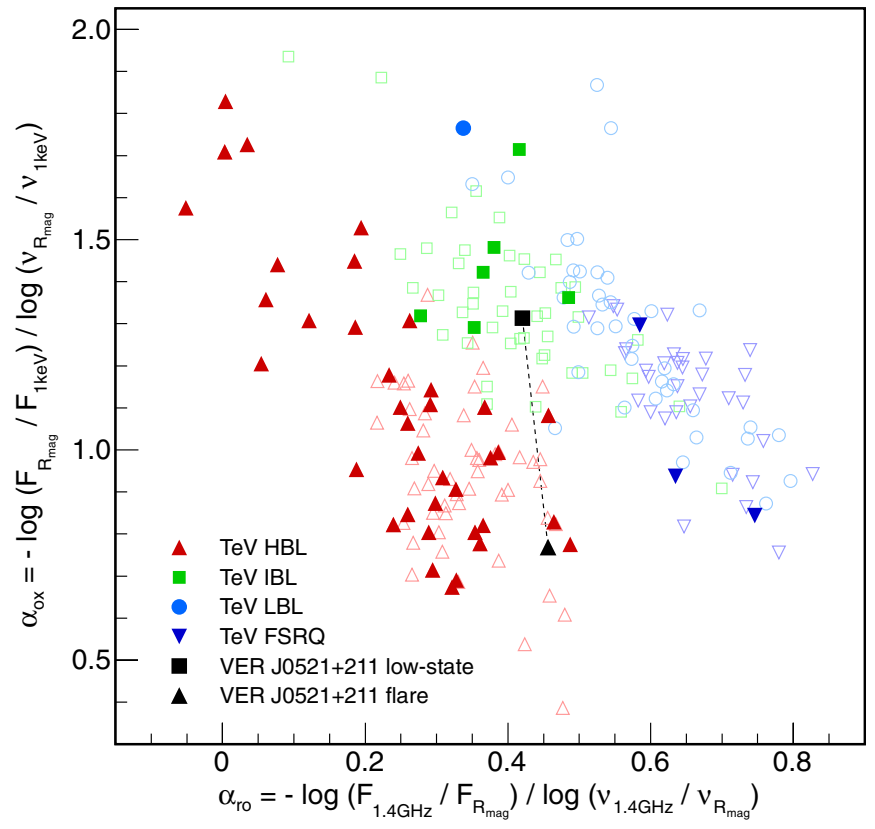

Figure 8. Spectral slopes of the synchrotron component for gamma-ray blazars The effective spectral indices $\alpha_{\text {ro }}$ and $\alpha_{\text {ox }}$ are defined in the usual way between $1.4 \mathrm{GHz}, 6590 \AA$, and $1 \mathrm{keV}$. SED classifications for the 47 known TeV blazars (filled markers) are taken from TeVCat (Wakely \& Horan 2008). Multiband fluxes are obtained from Massaro et al. (2009) with the exceptions of RX J0648.7+1516, HESS J1943+213, and MAGIC J2001+435 (Condon et al. 1998; Monet et al. 2003; Voges et al. 1999; Bassani et al. 2009). Empty markers show GeV-detected blazars from Nolan et al. (2012) present in Massaro et al. (2009). VER J0521+211 is pictured in a "low-state" (2009 October 22-30) showing a spectral shape characteristic of IBLs, and in "flare" (2009 November 27), when it shows HBL-like properties.

(A color version of this figure is available in the online journal.)

(Acciari et al. 2009), or PKS 1222+216 (Aleksić et al. 2011), have been detected at flux levels higher than 0.1 Crab during short periods of time ( $\leqslant 1$ week) and then quickly fall below instrument sensitivity. Despite being a relatively bright $\mathrm{TeV}$ source, VER J0521+211 was never identified as a TeV-blazar candidate before the VERITAS detection. Given its low Galactic latitude of -8.7 , VER J0521+211 was poorly characterized in the optical and X-ray bands and was not included in blazar catalogs, which are usually incomplete at low Galactic latitudes or avoid the Galactic plane altogether (e.g., Healey et al. 2008; Massaro et al. 2009), as diffuse radio emission, confusion with local radio sources, and heavy optical extinction make candidate blazars difficult to identify. Therefore, TeV-candidate catalogs (e.g., Costamante \& Ghisellini 2002; Donato et al. 2001) lacked sufficient multiwavelength information on VER J0521+211 and a firm identification as a blazar, required to predict its $\mathrm{TeV}$ flux.

\section{REDSHIFT UPPER LIMIT}

An upper limit on the redshift of VER J0521+211 can be derived from its TeV spectrum. Following the approach in Mazin \& Goebel (2007; see also Aharonian et al. 2006b; Mazin \& Raue 2007), the intrinsic TeV spectrum of VER J0521+211 is reconstructed from the VERITAS measured spectrum (Figure 2) by correcting for extragalactic background light absorption, assuming the density model of Domínguez et al. (2011), under varying assumptions of $z$. The intrinsic spectrum is then fit with a power law of the form $d N / d E \propto E^{-\Gamma^{\star}}$. Larger values of $z$ result in a harder reconstructed photon index $\Gamma^{\star}$. Classical leptonic emission models predict $\Gamma^{\star}>1.5$ (see discussion
Table 3

SED Modeling Parameters

\begin{tabular}{lcc}
\hline \hline Parameter & Symbol & Value \\
\hline Electron distribution & $L_{e}\left(\mathrm{erg} \mathrm{s}^{-1}\right)$ & $7.7 \times 10^{44}$ \\
Electron power & $\gamma_{\min }$ & $3.5 \times 10^{4}$ \\
Low-energy cutoff & $\gamma_{\max }$ & $2.0 \times 10^{6}$ \\
High-energy cutoff & $q_{e}$ & 3.0 \\
Injection index & $R_{b}(\mathrm{~cm})$ & $4.0 \times 10^{17}$ \\
Blob radius & $B(\mathrm{G})$ & 0.0025 \\
Magnetic field & $\Gamma$ & 30 \\
Bulk Lorentz factor & $\eta_{\mathrm{esc}}$ & 300 \\
Escape parameter & $z$ & 0.10 \\
Redshift (assumed) & & \\
\hline
\end{tabular}

in Aharonian et al. 2006b). Under this assumption, a redshift upper limit of $z<0.34$ is derived for VER J0521+211 at $95 \%$ confidence level. An even more conservative redshift upper limit can be obtained by allowing an intrinsic spectral index as hard as $\Gamma^{\star} \sim 0.7$, as suggested in Katarzyński et al. (2006; see also Aharonian et al. 2008; Sitarek \& Bednarek 2008). Under this less restrictive assumption of $\Gamma^{\star}>0.7$, the redshift of VER $\mathrm{J} 0521+211$ is constrained to $z<0.44$.

The redshift upper limits derived from the $\mathrm{TeV}$ spectrum of VER J0521+211 are in agreement with the recent measurement of $z=0.108$ based on a single optical spectral feature (Shaw et al. 2013).

\section{SPECTRAL ENERGY DISTRIBUTION}

After a successful identification of VER J0521+211 as a new $\mathrm{TeV}$ blazar, the available multiwavelength data were combined to construct an SED, shown in Figure 6. Most commonly accepted models attribute the low-energy emission component to synchrotron radiation by relativistic electrons in the jet magnetic field, and the high-energy to inverseCompton scattering of ambient photons off the same electron population (see, e.g., Maraschi et al. 1992).

The multiwavelength SED can be described with a one-zone synchrotron self-Compton (SSC) emission model as described in Böttcher et al. (2013). The model parameters are adjusted to describe the quasi-simultaneous spectral points obtained during the "low" emission state (2009 October 22-30). Models were tested assuming $z=0.05,0.1,0.15,0.2,0.25$, although only results for $z=0.1$ are discussed, being the assumption that most closely matches the tentative redshift of $z=0.108$.

The model parameters describing the low-state SED of VER $\mathrm{J} 0521+211$ are listed in Table 3, and show a slightly low magnetic field and large emitting region compared to other TeV blazars (Aliu et al. 2013). One-zone SSC models can only describe Compton-dominated systems $\left(R_{C} \gtrsim 1\right)$ like VER $\mathrm{J} 0521+211$ with very low magnetic fields, resulting in a strongly particle-dominated jet $\left(L_{B} / L_{e}<0.01 ; L_{B}\right.$ and $L_{e}$ being the magnetic and particle power in the jet, respectively). External Compton models (EC; Dermer \& Schlickeiser 1993) add a second population of low-energy photons where relativistic electrons inverse-Compton scatter, increasing the level of highenergy emission, with jet energetics close to equipartition $\left(L_{B} / L_{e} \sim 1\right)$. However, given the scarcity of simultaneous observations, particularly in the synchrotron component, an EC model applied to the SED of VER J0521+211 would be severely underconstrained. 


\section{SUMMARY AND CONCLUSIONS}

VERITAS detected a new TeV source: VER J0521+211, spatially associated with the radio and X-ray source RGB J0521.8+2112. Follow-up observations in the optical and X-ray bands unambiguously identify VER J0521+211 as a new blazar of the BL Lac type, displaying all the defining properties of blazars in radio, infrared, and optical wavelengths. The detected $\mathrm{TeV}$ emission is variable on daily timescales with an integral flux of $\sim 0.09-0.33$ Crab measured between 0.2 and $\sim 1 \mathrm{TeV}$, and a time-averaged spectrum compatible with a power law with photon index $\Gamma=3.44 \pm 0.20_{\text {stat }} \pm 0.30_{\text {syst }}$. During the observing campaign that covered 2009 October to 2010 January, VER J0521+211 transitioned to a high state on 2009 November 27 , when the nightly flux increased by a factor of $\sim 3$ in the $\mathrm{TeV}$ range and by a factor of $\sim 15$ in X-rays, compared to the observed baseline values. X-ray observations show a trend of spectral hardening with increasing flux, while no significant spectral variability was found at $\mathrm{TeV}$ energies.

Observations from radio to $\mathrm{X}$-ray frequencies show a multitude of evidence for a synchrotron origin of the emission from VER J0521+211 below a few keV, as expected for a blazar: a flat spectrum in the radio band with a turnover toward infrared frequencies, polarized emission in the optical band and in the $15 \mathrm{GHz}$ radio images from VLBA, and a nonthermal powerlaw spectrum in X-rays. The radio images also show electric polarization vectors perpendicularly aligned to the jet ridgeline, suggesting a relatively well-ordered magnetic field in the direction of the jet axis. The multiband spectral shape of the synchrotron component of VER J0521+211 is similar to that of known TeV IBLs. However, during the TeV and X-ray flare its synchrotron properties are closer to those of HBLs. Optical spectrometry with MDM could not be used to derive a redshift, although recent measurements suggest $z=0.108$. The TeV spectrum of VER J0521+211 constrains its redshift to $z<0.34$ under the assumption that the intrinsic $\mathrm{TeV}$ photon index of the source is $\Gamma^{\star}>1.5$, which is the limit obtained for standard leptonic emission models.

The high-energy emission from VER J0521+211 peaks in the gamma-ray band, between 10 and $200 \mathrm{GeV}$, and can be described with a leptonic one-zone SSC emission model. The resulting model parameters would indicate a relatively weak magnetic field of $\lesssim 0.01 \mathrm{G}$ and a particle-dominated jet. Similar objects have been modeled by adding an EC component to the SSC emission (Acciari et al. 2009; Abdo et al. 2011a), or considering a structured jet with a fast-moving spine and a slower outer layer (Anderhub et al. 2009). These models could presumably describe the SED of VER J0521+211, and generally reach solutions closer to equipartition. However, being more complex than SSC emission, SSC+EC and structured jet models have more free parameters, and would be underconstrained given the data available for VER J0521+211.

Because of its low Galactic latitude, VER J0521+211 observations were not triggered by its $\mathrm{X}$-ray properties like most TeV-candidate blazars, but by a cluster of $E>30 \mathrm{GeV}$ photons detected in the Fermi-LAT public data released after the first year of observations. Selection criteria based on Fermi-LAT data have been successful in identifying other new TeV blazars (Aleksić et al. 2011; Aliu et al. 2012; Zech et al. 2011), particularly at low Galactic latitudes where selections based on radio and X-ray data are less powerful due to Galactic extinction. The detection of VER J0521+211 adds to previous results that have demonstrated the strength of $\mathrm{GeV}$ band (e.g., Mukherjee et al. 2000; Vandenbroucke et al. 2010; Kara et al. 2012) and
TeV-band observations (e.g., Mariotti 2010; Aliu et al. 2011; Abramowski et al. 2011) as a tool to identify blazars located behind the Galactic plane.

With a TeV flux between 0.09 and 0.33 Crab, VER J0521+211 ranks among the brightest known $\mathrm{TeV}$ blazars, and can be detected with current ground-based Cherenkov telescopes in less than $1 \mathrm{hr}$ exposure. Assuming a redshift of 0.108 , the TeV luminosity of VER J0521+211 is $L_{>0.2 \mathrm{TeV}} \sim 2.4 \times 10^{44} \mathrm{erg} \mathrm{s}^{-1}$, larger than that of the "classical" northern TeV blazars (Mrk 421, Mrk 501, and 1ES 1959+650), which sample the low-luminosity end of the population of TeV blazars (Şentürk et al. 2013). Given the observed variability and its bright $\mathrm{TeV}$ flux, future multiwavelength observations of VER J0521+211 will be able to extend the detailed time-resolved spectral modeling available for nearby HBLs (Krawczynski et al. 2004; Acciari et al. 2011; Abdo et al. 2011b) to a more luminous non-HBL blazar.

VERITAS is supported by grants from the U.S. Department of Energy Office of Science, the U.S. National Science Foundation, and the Smithsonian Institution, by NSERC in Canada, by Science Foundation Ireland (SFI 10/RFP/AST2748), and by STFC in the UK. We acknowledge the excellent work of the technical support staff at the Fred Lawrence Whipple Observatory and at the collaborating institutions in the construction and operation of the instrument.

The Fermi-LAT Collaboration acknowledges support from a number of agencies and institutes for both development and the operation of the LAT as well as scientific data analysis. These include NASA and DOE in the United States, CEA/Irfu and IN2P3/CNRS in France, ASI and INFN in Italy, MEXT, KEK, and JAXA in Japan, and the K. A. Wallenberg Foundation, the Swedish Research Council, and the National Space Board in Sweden. Additional support from INAF in Italy and CNES in France for science analysis during the operations phase is also gratefully acknowledged.

M.E. acknowledges support from the NASA grants NNX10AP66G and NNX12AJ30G. Y.Y.K. was supported in part by the Russian Foundation for Basic Research (projects 1102-00368 and 12-02-33101), the basic research program "Active Processes in Galactic and Extragalactic Objects" of the Physical Sciences Division of the Russian Academy of Sciences, and the Dynasty Foundation. M.B. acknowledges support by the South African Department of Science and Technology through the National Research Foundation under NRF SARChI Chair grant No. 64789.

This research has made use of data from the MOJAVE database that is maintained by the MOJAVE team (Lister et al. 2009a). The MOJAVE project is supported under NASA-Fermi grants NNX08AV67G and 11-Fermi11-0019. The authors thank Julie Skinner for obtaining, as a target of opportunity, the first MDM spectrum of RGB J0521.8+2112 used in this paper, and Talvikki Hovatta for providing the OVRO radio data. Observations at Steward Observatory were supported by the NASA Fermi Guest Investigator Program grant NNX09AU10G. Finally, the authors thank the Swift team for accepting and carefully scheduling the target of opportunity observations of VER J0521+211 that were used in the paper and for support from the Swift Guest Investigator program, NASA grant NNX10AF89G.

\section{REFERENCES}

Abdo, A. A., Ackermann, M., Ajello, M., et al. 2009, ApJ, 707, 1310 Abdo, A. A., Ackermann, M., Ajello, M., et al. 2010a, ApJS, 188, 405 
Abdo, A. A., Ackermann, M., Ajello, M., et al. 2010b, ApJ, 715, 429 Abdo, A. A., Ackermann, M., Ajello, M., et al. 2011a, ApJ, 726, 43 Abdo, A. A., Ackermann, M., Ajello, M., et al. 2011b, ApJ, 727, 129 Abdo, A. A., Ackermann, M., Ajello, M., et al. 2011c, ApJ, 730, 101 Abramowski, A., Acero, F., Aharonian, F., et al. 2011, A\&A, 529, A49

Abramowski, A., Acero, F., Akhperjanian, A. G., et al. 2013, A\&A, 552, A118

Acciari, V. A., Aliu, E., Aune, T., et al. 2009, ApJ, 707, 612

Acciari, V. A., Aliu, E., Arlen, T., et al. 2011, ApJ, 738, 25

Acciari, V. A., Beilicke, M., Blaylock, G., et al. 2008, ApJ, 679, 1427

Ackermann, M., Ajello, M., Albert, A., et al. 2012, ApJS, 203, 4

Ackermann, M., Ajello, M., Allafort, A., et al. 2013, submitted to ApJS (arXiv:1306.6772)

Aharonian, F., Akhperjanian, A., Barrio, J., et al. 2001, A\&A, 370, 112

Aharonian, F., Akhperjanian, A. G., Bazer-Bachi, A. R., et al. 2006a, ApJ, 636, 777

Aharonian, F., Akhperjanian, A. G., Bazer-Bachi, A. R., et al. 2006b, Natur, 440, 1018

Aharonian, F. A., Khangulyan, D., \& Costamante, L. 2008, MNRAS, 387,1206

Albert, J., Aliu, E., Anderhub, H., et al. 2008a, ApJ, 674, 1037

Albert, J., Aliu, E., Anderhub, H., et al. 2008b, Sci, 320, 1752

Aleksić, J., Antonelli, L. A., Antoranz, P., et al. 2011, ApJL, 730, L8

Aliu, E., Archambault, S., Arlen, T., et al. 2012, ApJ, 750, 94

Aliu, E., Archambault, S., Arlen, T., et al. 2013, ApJ, submitted

Aliu, E., Aune, T., Beilicke, M., et al. 2011, ApJ, 742, 127

Anderhub, H., Antonelli, L. A., Antoranz, P., et al. 2009, ApJL, 704, L129

Atwood, W. B., Abdo, A. A., Ackermann, M., et al. 2009, ApJ, 697, 1071

Bassani, L., Landi, R., Masetti, N., et al. 2009, MNRAS, 397, L55

Beasley, A. J., Gordon, D., Peck, A. B., et al. 2002, ApJS, 141, 13

Berge, D., Funk, S., \& Hinton, J. 2007, A\&A, 466, 1219

Böttcher, M., Reimer, A., Sweeney, K., \& Prakash, A. 2013, ApJ, 768, 54

Burrows, D. N., Hill, J. E., Nousek, J. A., et al. 2005, SSRv, 120, 165

Cohen, A. S., Lane, W. M., Cotton, W. D., et al. 2007, AJ, 134, 1245

Condon, J. J., Cotton, W. D., Greisen, E. W., et al. 1998, AJ, 115, 1693

Costamante, L., \& Ghisellini, G. 2002, A\&A, 384, 56

Cutri, R. M., Skrutskie, M. F., van Dyk, S., et al. 2003, The IRSA 2MASS All-Sky Point Source Catalog, NASA/IPAC Infrared Science Archive, http://vizier.cfa.harvard.edu/viz-bin/VizieR?-source=11/246

Dermer, C. D., \& Schlickeiser, R. 1993, ApJ, 416, 458

Domínguez, A., Primack, J. R., Rosario, D. J., et al. 2011, MNRAS, 410, 2556

Donato, D., Ghisellini, G., Tagliaferri, G., \& Fossati, G. 2001, A\&A, 375, 739

Drake, A. J., Djorgovski, S. G., Mahabal, A., et al. 2009, ApJ, 696, 870

Fomin, V. P., Stepanian, A. A., Lamb, R. C., et al. 1994, APh, 2, 137

Gehrels, N., Chincarini, G., Giommi, P., et al. 2004, ApJ, 611, 1005

Gregory, P. C., Scott, W. K., Douglas, K., \& Condon, J. J. 1996, ApJS, 103, 427

Healey, S. E., Romani, R. W., Cotter, G., et al. 2008, ApJS, 175, 97

Hillas, A. M. 1985, ICRC, 3, 445

Hillas, A. M., Akerlof, C. W., Biller, S. D., et al. 1998, ApJ, 503, 744

Hinton, J. A. 2004, NewAR, 48, 331

Holder, J., Acciari, V. A., Aliu, E., et al. 2008, in Proc. 4th Int. Meeting on High Energy Gamma-Ray Astronomy, AIP Conf. Ser. 1085, ed. F. A. Aharonian, W. Hofmann, \& F. Rieger (Melville, NY: AIP), 657

Holder, J., Atkins, R. W., Badran, H. M., et al. 2006, APh, 25, 391

Jackson, B., Scargle, J. D., Barnes, D., et al. 2005, ISPL, 12, 105
Jackson, N., Battye, R. A., Browne, I. W. A., et al. 2007, MNRAS, 376, 371

Kalberla, P. M. W., Burton, W. B., Hartmann, D., et al. 2005, A\&A, 440, 775

Kara, E., Errando, M., Max-Moerbeck, W., et al. 2012, ApJ, 746, 159

Katarzyński, K., Ghisellini, G., Tavecchio, F., Gracia, J., \& Maraschi, L. 2006, MNRAS, 368, L52

Kellermann, K. I., Sramek, R., Schmidt, M., Shaffer, D. B., \& Green, R. 1989, AJ, 98, 1195

Kovalev, Y. Y. 2009, ApJL, 707, L56

Krawczynski, H., Hughes, S. B., Horan, D., et al. 2004, ApJ, 601, 151

Laurent-Muehleisen, S. A., Kollgaard, R. I., Ryan, P. J., et al. 1997, A\&AS, 122,235

Li, T.-P., \& Ma, Y.-Q. 1983, ApJ, 272, 317

Lister, M. L., Aller, H. D., Aller, M. F., et al. 2009a, AJ, 137, 3718

Lister, M. L., Cohen, M. H., Homan, D. C., et al. 2009b, AJ, 138, 1874

Lister, M. L., \& Homan, D. C. 2005, AJ, 130, 1389

Maraschi, L., Ghisellini, G., \& Celotti, A. 1992, ApJL, 397, L5

Mariotti, M. 2010, ATel, 2753, 1

Massaro, E., Giommi, P., Leto, C., et al. 2009, A\&A, 495, 691

Massaro, F., D’Abrusco, R., \& Paggi, A. 2012a, ATel, 4128, 1

Massaro, F., D’Abrusco, R., Tosti, G., et al. 2012b, ApJ, 752, 61

Massaro, F., Tramacere, A., Cavaliere, A., Perri, M., \& Giommi, P. 2008, A\&A, 478, 395

Mazin, D., \& Goebel, F. 2007, ApJL, 655, L13

Mazin, D., \& Raue, M. 2007, A\&A, 471, 439

Meyer, E. T., Fossati, G., Georganopoulos, M., \& Lister, M. L. 2011, ApJ, 740, 98

Meyer, E. T., Fossati, G., Georganopoulos, M., \& Lister, M. L. 2012, ApJL, 752, L4

Monet, D. G., Levine, S. E., Canzian, B., et al. 2003, AJ, 125, 984

Mukherjee, R., Gotthelf, E. V., Halpern, J., \& Tavani, M. 2000, ApJ, 542, 740

Neronov, A., Semikoz, D., \& Vovk, I. 2010, A\&A, 519, L6

Nolan, P. L., Abdo, A. A., Ackermann, M., et al. 2012, ApJS, 199, 31

Ong, R. A. 2009a, ATel, 2260, 1

Ong, R. A. 2009b, ATel, 2301, 1

Perkins, J. S., Maier, G., \& The VERITAS Collaboration, 2009, in Proc. 2nd Fermi Symp., ed. D. J. Thompson (Menlo Park, CA: SLAC), eConf C0911022

Richards, J. L., Max-Moerbeck, W., Pavlidou, V., et al. 2011, ApJS, 194, 29

Scargle, J. D. 1998, ApJ, 504, 405

Schlafly, E. F., \& Finkbeiner, D. P. 2011, ApJ, 737, 103

Schmidt, G. D., Stockman, H. S., \& Smith, P. S. 1992, ApJL, 398, L57

Şentürk, G. D., Errando, M., Böttcher, M., \& Mukherjee, R. 2013, ApJ, 764, 119

Shaw, M. S., Romani, R. W., Cotter, G., et al. 2013, ApJ, 764, 135

Sitarek, J., \& Bednarek, W. 2008, MNRAS, 391, 624

Urry, C. M., \& Padovani, P. 1995, PASP, 107, 803

Vandenbroucke, J., Buehler, R., Ajello, M., et al. 2010, ApJL, 718, L166

Voges, W., Aschenbach, B., Boller, T., et al. 1999, A\&A, 349, 389

Wakely, S. P., \& Horan, D. 2008, ICRC, 3, 1341

Wardle, J. F. C., \& Kronberg, P. P. 1974, ApJ, 194, 249

Weinstein, A., \& for the VERITAS Collaboration, 2009, in Proc. 2nd Fermi Symp., ed. D. J. Thompson (Menlo Park, CA: SLAC), eConf C0911022

White, R. L., \& Becker, R. H. 1992, ApJS, 79, 331

Wright, E. L., Eisenhardt, P. R. M., Mainzer, A. K., et al. 2010, AJ, 140, 1868

Zech, A., Behera, B., Becherini, Y., et al. 2011, arXiv:1105.0840 